\title{
Surgical outcome for hypertrophic cardiomyopathy patients with extreme interventricular septal thickness: a propensity score matched study
}

\author{
Changwei Ren, Shengwei Wang, Jianbo Yu, Hongchang Guo, Hehe Ma, Zhipeng Wei, Kemin Liu, \\ Yongqiang Lai \\ Department of Cardiovascular Surgery Center, Beijing Anzhen Hospital, Capital Medical University, Beijing Institute of Heart, Lung and Blood \\ Vascular Diseases, Beijing, China \\ Contributions: (I) Conception and design: Y Lai; (II) Administrative support: Y Lai; (III) Provision of study materials or patients: Y Lai, J Yu; (IV) \\ Collection and assembly of data: S Wang, H Guo, K Liu, H Ma, C Ren; (V) Data analysis and interpretation: C Ren, S Wang; (VI) Manuscript \\ writing: All authors; (VII) Final approval of manuscript: All authors. \\ Correspondence to: Yongqiang Lai, MD. No.2, Anzhen Road, Chaoyang District Beijing 100029, China. Email: yongqianglai@yahoo.com.
}

\begin{abstract}
Background: Hypertrophic cardiomyopathy (HCM) with extreme interventricular septal thickness (IVST) is associated with a higher incidence of adverse cardiovascular events. However, the results of these patients who underwent septal myectomy are unclear.

Methods: We studied $47 \mathrm{HCM}$ patients with IVST $\geq 30 \mathrm{~mm}$ who underwent septal myectomy between 2011 and 2018 in Anzhen Hospital. After a 2:1 propensity score matching, the study cohort included 141 patients and 94 patients with IVST $<30 \mathrm{~mm}$.

Results: Patients with IVST $\geq 30 \mathrm{~mm}$ had a longer clinical course of disease, high incidence of syncope, palpitation, and moderate or severe mitral regurgitation. After a mean follow-up of $34.0 \pm 21.3$ months, 6 patients died, including 5 with IVST $\geq 30 \mathrm{~mm}$ and 1 with IVST $<30 \mathrm{~mm}$. The 5 -year survival free of allcause mortality was significantly higher in patients with IVST $\geq 30 \mathrm{~mm}$ than in those with IVST $<30 \mathrm{~mm}$ (98.9\% vs. 85.7\%, $\mathrm{P}=0.03$ ). Multivariable Cox analysis revealed that IVST $\geq 30 \mathrm{~mm}$ (HR: $1.12,95 \%$ CI: 1.01-1.25, $\mathrm{P}=0.028$ ) was an independent risk factor for all-cause mortality. Meanwhile, left ventricular end diastole diameter (HR: 0.72, 95\% CI: 0.54-0.97, P=0.031) and age (HR: 0.91, 95\% CI: 0.83-0.99, P=0.025) were also independent risk factors for all-cause mortality in this special cohort. Furthermore, the incidence of NYHA class III or IV was significantly higher in patients with IVST $\geq 30 \mathrm{~mm}$.

Conclusions: The surgical outcome was poor in a matched cohort of HCM patients with IVST $\geq 30 \mathrm{~mm}$, which was mainly reflected by mortality and the incidence of NYHA class III or IV.
\end{abstract}

Keywords: Hypertrophic cardiomyopathy (HCM); interventricular septal thickness (IVST); septal myectomy

Submitted Apr 18, 2020. Accepted for publication Sep 04, 2020.

doi: $10.21037 /$ jtd-20-1705

View this article at: http://dx.doi.org/10.21037/jtd-20-1705

\section{Introduction}

Hypertrophic cardiomyopathy (HCM) is the most common inherited heart disease, with a prevalence of $0.02 \%$ in the general population. It is an important cause of sudden cardiac death (SCD) (1). Approximately $2 / 3$ of patients who have a left ventricular outflow tract obstruction (LVOTO) need surgery to control the symptoms and reduce the risk of SCD. Septal myectomy is the standard treatment for patients who have a good prognosis $(2,3)$. There are many factors associated with SCD in these patients, including maximum left ventricular wall thickness $(4,5)$. HCM patients with extreme interventricular septal thickness (IVST) may represent a special cohort. However, most of these studies are all based on HCM patients, both 
obstructed and non-obstructed (6-8). Few studies have reported the surgical outcomes of patients with extreme IVST who underwent septal myectomy. Therefore, in the present study, we retrospectively studied this special cohort and compared the survival rate with patients matched by age, sex, and body mass index.

We present the following article in accordance with the STROBE reporting checklist (available at http://dx. doi. org/10. 21037/jtd-20-1705).

\section{Methods}

\section{Study population}

We retrospectively studied 47 hypertrophic obstructive cardiomyopathy (HOCM) patients with IVST $\geq 30 \mathrm{~mm}$ and 94 patients with IVST $<30 \mathrm{~mm}$ who underwent surgical treatment at Anzhen Hospital in Beijing between 2011 and 2018. This was a single-center retrospective study. The Control Group (patients with IVST $<30 \mathrm{~mm}$, $\mathrm{N}=94$ ) were enrolled from the same center and the IVST $\geq 30 \mathrm{~mm}$ was matched in a ratio of 2:1 based on age, sex, and body mass index and were randomly selected from subjects who underwent septal myectomy during the same period. The diagnostic criteria and surgical indications of HCM were consistent with the 2011 American Heart Association/American College of Cardiology guidelines and the 2014 European Society of Cardiology guidelines, which mainly include unexplained septal hypertrophy with a thickness $>15 \mathrm{~mm}$. The indications for septal myectomy were (I) severe symptoms or syncope or near-syncope despite optimal medical therapy, (II) LV outflow tract (LVOT) gradient $>50 \mathrm{mmHg}$ at rest or with provocation.

All patients signed informed consent forms, including those for their biomarker analysis and clinical data, prior to enrollment. The study was approved by the Ethics Committee of Anzhen Hospital (Ethic committee study number: 2020034X). All procedures were conducted in accordance with the ethical principles stated in the Declaration of Helsinki (as revised in 2013).

\section{Echocardiography}

Transthoracic echocardiography was performed in each patient using an E9 ultrasound system. Echocardiographic examinations were performed by the same physician. The diameter of the cardiac chambers was expressed as the maximum value of the anteroposterior diameter in the cardiac cycles. The diameter of the ascending aorta was approximately $4 \mathrm{~cm}$ above that of the aortic valve during diastole. The thicknesses of the IVS and ventricular wall were determined during diastole. Besides the maximum thickness, the representative thickness of the IVS, which is usually the thickness of the point $25 \mathrm{~mm}$ under the right coronary sinus nadir, was also recorded to indicate the overall thickness. The LVOT gradient was calculated using the simplified Bernoulli equation. The measurements of the left ventricular ejection fraction were determined according to the American Society of Echocardiography recommendations (9).

\section{Cardiac surgery}

All the patients underwent the extended Morrow procedure. First, a standard median sternotomy was performed. Cardiopulmonary bypass was then performed using ascending aortic cannulation and bicaval cannulation. Myocardial protection was achieved with the antegrade (HTK). The resection ranges were as follows: the upper end was located approximately $4 \mathrm{~mm}$ below the aortic ring; the lower end was extended toward the apex; from the right side, the myectomy was started slightly rightward to the nadir of the right aortic cusp, and to the left, resection was terminated near the mitral anterior commissure. Elongated leaflets were not routinely folded, unless severe regurgitation or systolic anterior motion remained after resection. The related operations were appropriately performed based on preoperative evaluation and intraoperative exploration. Detailed information has been described in our previous study (10).

\section{Follow-up}

The clinical status of the study population was obtained through telephone interviews with the patients or their family members at least once a year after surgery. Patients who died were treated as endpoints, and the followup time was defined with regard to the day the patient died. The last follow-up of the survivors was conducted in December 2019. The clinical endpoint in this study was defined as all-cause death, including sudden cardiac death (SCD), death related to congestive heart failure and other cardiovascular diseases, or noncardiac death. In addition, all patients in the present study completed the follow-up. 


\section{Statistical analysis}

In the present study, we performed a propensity score match for the variables according to the presence of extreme IVST ( $\geq 30 \mathrm{~mm}$ ): age, sex, and BMI. Matching was performed using the nearest neighbor method, assigning patients with diabetes and without diabetes in a $1: 2$ ratio, with a 0.2 caliper width. The equalization test after matching is shown in Table S1 and Figure S1. The Kolmogorov-Smirnov test was used to assess the normal distribution of continuous variables. The results are expressed as mean \pm standard deviation, median (interquartile range), or percentage, as appropriate. The Student $t$-test for independent samples and the MannWhitney $\mathrm{U}$ test were used to compare continuous variables, and the $\chi^{2}$ or Fisher exact test was used to compare nominal variables, as appropriate. The Kaplan-Meier method was used to calculate survival free from the worse clinical outcomes. A log-rank test was used to compare survival curves among the different patient groups. Univariable and multivariable Cox regression analyses were used to assess the association of individual variables with all-cause death. Age, sex, body mass index, and variables with $\mathrm{P}<0.1$ on univariate analysis were entered into a multivariate analysis. All the reported probability values were two-tailed, and $\mathrm{P}<0.05$ was considered statistically significant. SPSS version 24.0 Statistical software (IBM) and Prism GraphPad 7.0 (GraphPad Software Inc., La Jolla, CA, USA) were used for calculations and illustrations, respectively.

\section{Results}

\section{Baseline patient characteristics}

A total of 141 HCM patients were included in the study, including 47 patients with IVST $\geq 30 \mathrm{~mm}$. We compared the baseline characteristics of the patients with an IVST $<30 \mathrm{~mm}$. The clinical course of disease $(7.2 \pm 6.9 \mathrm{vs}$. $4.2 \pm 4.0, \mathrm{P}=0.008)$ was significantly longer in patients with IVST $\geq 30 \mathrm{~mm}$, and the serum BNP level was also high in these patients. There were more symptoms, including syncope and palpitation, in patients with IVST $\geq 30 \mathrm{~mm}$. Importantly, the incidence of non-sustained ventricular tachycardia (NSVT) was higher in patients with IVST $\geq 30 \mathrm{~mm}$. In addition, moderate or severe mitral regurgitation, left atrium, left ventricular end diastole diameter, and IVST were also significantly different between the two groups. Detailed information is described in Table 1.

\section{Perioperative data}

Perioperative data between the two groups are described in Table 2. There was no difference in mitral valve procedure, CABG, Cox Maze procedure, cardiopulmonary bypass time, aortic cross-clamping time, postoperative ventilation time, and postoperative hospital stay between the two groups. However, the ICU stay time $(47.1 \pm 31.4$ vs. $36.3 \pm 23.9$, $\mathrm{P}=0.03)$ and the proportion of tricuspid valvuloplasty $(8.5 \%$ vs. $0.0 \%, \mathrm{P}=0.05$ ) were significantly different between IVST $\geq 30 \mathrm{~mm}$ and IVST $<30 \mathrm{~mm}$. In addition, the postoperative LVOT gradient was not different between the two groups.

\section{Clinical and echocardiographic data at last follow-up}

After a mean follow-up of $34.0 \pm 21.3$ months, the clinical presentation and echocardiographic data showed a significant improvement. Compared to the baseline data, the proportion of patients with NYHA class III or IV and Max LVOT gradient $>30 \mathrm{mmHg}$ decreased significantly. However, the proportion of patients with NYHA class III or IV was high in patients with IVST $\geq 30 \mathrm{~mm}$ at the latest follow-up (Table 3).

\section{Survival from all-cause death}

After a mean follow-up of $34.0 \pm 21.3$ months, a total of 6 patients died. Of these, 1 death was related to lung cancer and 5 were cardiovascular deaths, including 4 sudden cardiac deaths and 1 death related to heart failure. The Kaplan-Meier lifetime analysis showed a significant difference in the survival free from all-cause death between the two groups (98.9\% vs. $85.7 \%, \mathrm{P}=0.04$, Figure 1). Next, we performed univariable and multivariable Cox regression analyses to investigate the risk factors of all-cause death. After adjustment for age, sex, and BMI, IVST $\geq 30 \mathrm{~mm}$ was an independent risk factor for all-cause death (HR: 1.12, 95\% CI: $1.01-1.25, \mathrm{P}=0.028$ ). In addition, LVEDD (HR: 0.72, 95\% CI: 0.54-0.97, P=0.031) and age (HR: 0.91, 95\% CI: $0.83-0.99, \mathrm{P}=0.025$ ) were also risk factors for all-cause death in this special HCM cohort (Table 4).

\section{Discussion}

In our study, we report the surgical outcomes of HCM patients with extreme IVST. Important findings from our study comparing septal myectomy for extreme IVST patients was that overall survival of propensity-matched 
Table 1 Baseline patient characters

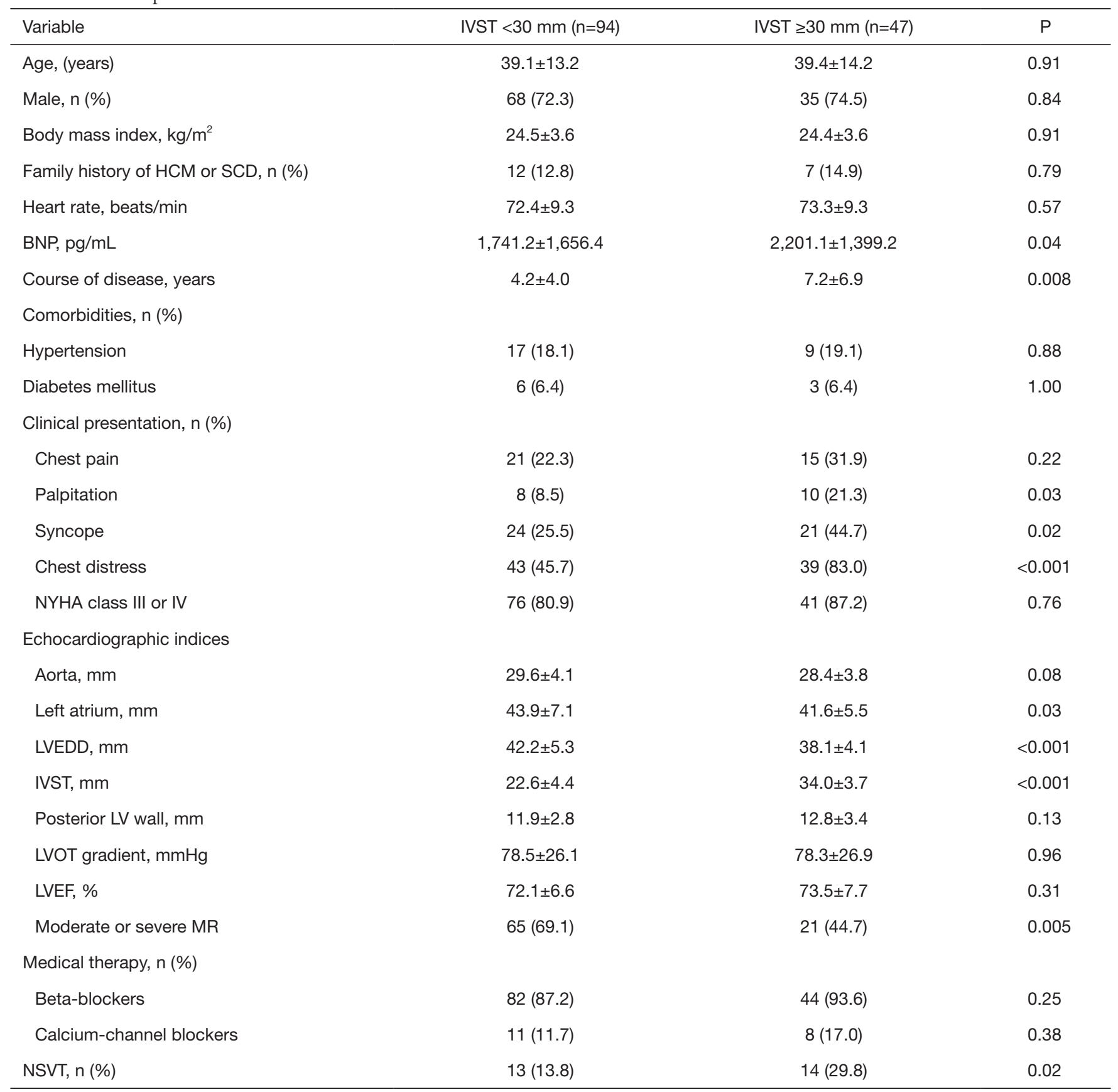

Values are presented as percentage, mean $\pm \mathrm{SD}$, or median (interquartile range) when appropriate. IVST, interventricular septal thickness; HCM, hypertrophic myocardiopathy; SCD, sudden cardiac death; NYHA, New York Heart Association; BNP, brain natriuretic peptide; LVEF, left ventricular ejection fraction; LVEDD, left ventricular end diastole diameter; LVOTG, left ventricular outflow tract gradient;

patients was significantly lower in this special cohort, and the proportion of patients with NYHA class III or IV was high at the latest follow-up. Furthermore, left ventricular end-diastolic diameter and young age were also associated with a poor prognosis in patients with extreme IVST in a matched cohort.

Relevant guidelines have pointed out that IVST $\geq 30 \mathrm{~mm}$ is an important risk factor for SCD in HCM 
Table 2 Perioperative data between two groups

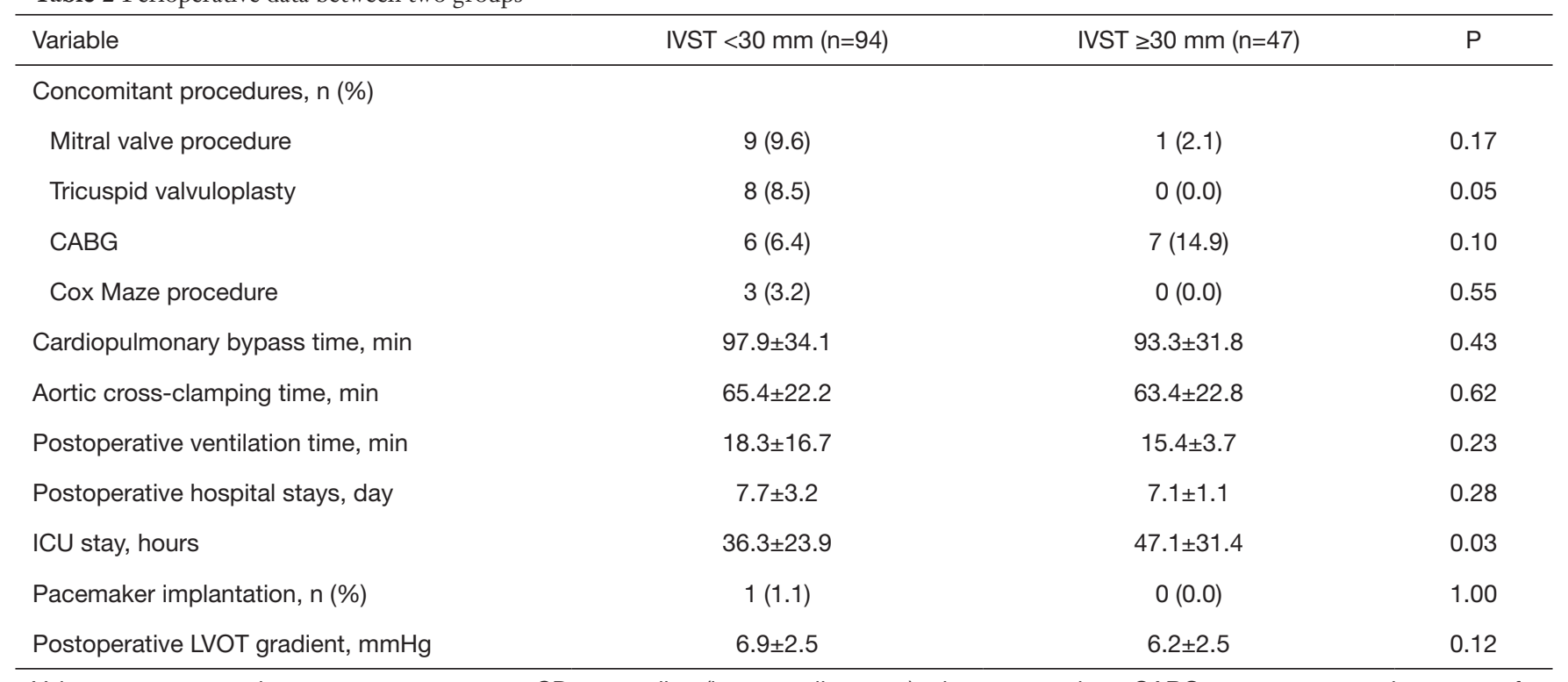

Values are presented as percentage, mean $\pm \mathrm{SD}$, or median (interquartile range) when appropriate. CABG, coronary artery bypass graft; ICU, intensive care unit.

Table 3 Clinical and Echocardiographic data at last-follow-up visit

\begin{tabular}{|c|c|c|c|}
\hline Variables & IVST $<30 \mathrm{~mm}(\mathrm{n}=94)$ & IVST $\geq 30 \mathrm{~mm}(\mathrm{n}=47)$ & $P$ \\
\hline \multicolumn{4}{|c|}{ NYHA class III or IV, n (\%) } \\
\hline Baseline & $76(80.9)$ & $41(87.2)$ & 0.76 \\
\hline Last follow-up & $2(2.1)$ & $6(12.8)$ & 0.02 \\
\hline \multicolumn{4}{|c|}{ Max LVOT gradient >30 mmHg, $\mathrm{n}(\%)$} \\
\hline Baseline & $94(100.0)$ & $47(100.0)$ & 1.00 \\
\hline Last follow-up & $8(8.5)$ & $3(6.4)$ & 0.75 \\
\hline \multicolumn{4}{|l|}{ IVST, mm } \\
\hline Baseline & $22.6 \pm 4.4$ & $34.0 \pm 3.7$ & $<0.001$ \\
\hline Baseline & $11.9 \pm 2.8$ & $12.8 \pm 3.4$ & 0.13 \\
\hline Last follow-up & $10.9 \pm 2.3$ & $11.9 \pm 3.1$ & 0.06 \\
\hline \multicolumn{4}{|l|}{ LVEDD, mm } \\
\hline Baseline & $42.2 \pm 5.3$ & $38.1 \pm 4.1$ & $<0.001$ \\
\hline Last follow-up & $44.6 \pm 4.6$ & $44.5 \pm 6.6$ & 0.96 \\
\hline
\end{tabular}

Values are presented as percentage, mean \pm SD, or median (interquartile range) when appropriate. NYHA, New York Heart Association; IVST, interventricular septal thickness; LVOT, left ventricular outflow tract gradient; LVEDD, left ventricular end diastole diameter. 
patients $(4,5)$. Recently, one study reported the impact of effective management strategies on patients with extreme IVST in HCM patients. They concluded that this special cohort is at increased arrhythmic sudden death risk, and progressive heart failure secondary to outflow obstruction was reversible after septal myectomy (6). However, they did not compare the mortality of extreme IVST after septal myectomy with a matched population. In addition, another study compared the survival rate after septal myectomy among three groups divided by the degree of IVST. The

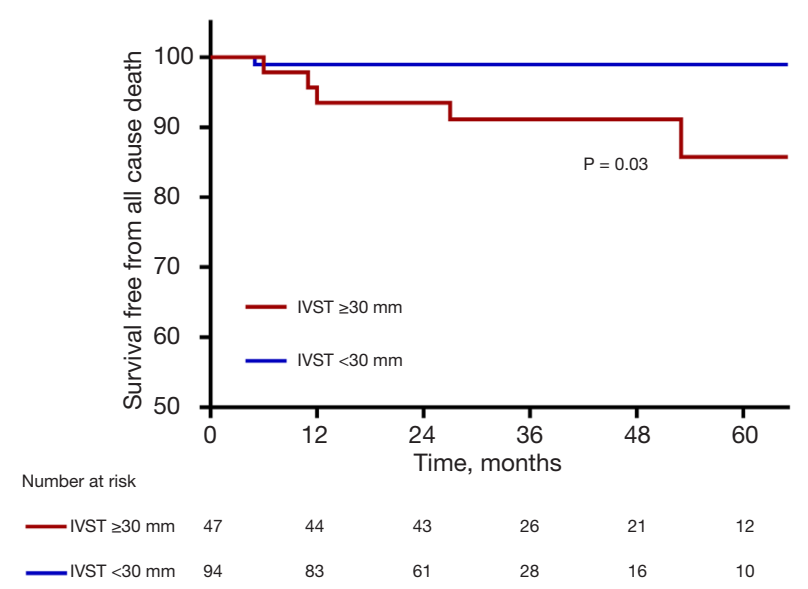

Figure 1 Kaplan-Meier analysis of survival free from all cause death between two groups. IVST, interventricular septal thickness. results showed that there was no difference in mortality among the different groups, even though the most extreme IVST was $21 \mathrm{~mm}$ in their study (11). None of these studies compared survival rates in patients with IVST $\geq 30 \mathrm{~mm}$ with survival rates in those matched patients with IVST $<30 \mathrm{~mm}$.

In the present study, we found that extreme IVST (IVST $\geq 30 \mathrm{~mm}$ ) of hypertrophic obstructive cardiomyopathy $(\mathrm{HOCM})$ patients was an independent risk factor for allcause death after adjusting for age, sex, and body mass index. In other words, for patients with this special phenotype, the prognosis is still relatively poor even after surgery. The clinical course of the disease was long in patients with IVST $\geq 30 \mathrm{~mm}$, which may reflect a more serious pathological change (12). In addition, the diameter of the left atrium and left ventricular end diastole were also significantly larger in patients with IVST $\geq 30 \mathrm{~mm}$. Previous studies have reported that both parameters are markers of disease severity in HCM (13-15). In addition, the proportion of moderate or severe mitral regurgitation was high in patients with IVST $<30 \mathrm{~mm}$; however, the mitral valve procedure during operation was similar between the two groups. Moreover, the incidence of NSVT was also significantly high in patients with IVST $\geq 30 \mathrm{~mm}$. Many studies have reported that NSVT was associated with SCD, cardiovascular death, and all-cause death in patients with HCM (16-18). Univariable analysis in our study showed that NSVT was related to all-cause death in this study cohort; however, multivariate analysis was not statistically significant. The

Table 4 Multivariate cox proportional Hazards Models for the all cause death

\begin{tabular}{llll}
\hline \multirow{2}{*}{ Variable } & \multicolumn{1}{c}{ Univariable } & & Multivariate \\
\cline { 2 - 3 } Age & $\mathrm{RR}(95 \% \mathrm{Cl})$ & $\mathrm{P}$ & $\mathrm{RR}(95 \% \mathrm{Cl})$ \\
Male & $0.89(0.82-0.98)$ & 0.01 & $0.91(0.83-0.99)$ \\
Body mass index & $1.62(0.18-14.49)$ & 0.67 & 0.025 \\
NT-Pro BNP & $1.01(0.78-1.31)$ & 0.94 & \\
NYHA class & $1.00(1.00-1.001)$ & 0.04 & $0.72(0.54-0.97)$ \\
LVEDD, mm & $7.19(1.37-37.76)$ & 0.02 & $1.12(1.01-1.25)$ \\
IVST, mm & $0.80(0.68-0.96)$ & 0.01 & 0.031 \\
LVEF, \% & $1.18(1.05-1.32)$ & 0.004 & \\
ICU stays, hours & $1.15(1.007-1.31)$ & 0.04 & \\
NSVT & $1.02(1.001-1.03)$ & 0.03 & \\
\hline
\end{tabular}

RR, relative risk; NYHA, New York Heart Association; LVEDD, left ventricular end diastole diameter; IVST, interventricular septal thickness; LVEF, left ventricular ejection fraction; ICU, intensive care unit. 
small number of patients, the matched cohort of the study, and relatively short-term follow-up might be associated with the present results. Furthermore, the time of ICU stay was long in patients with extreme IVST, which means that these patients need more time to return to a stable level.

The present results might provide some information about the future therapy for this special cohort with extreme IVST. On the one hand, for extreme IVST of HOCM patients, we should pay more attention and they should be followed up more closely, with regular Holter and echocardiography monitoring (19). On the other hand, if these patients experience syncope or severe arrhythmia events after septal myectomy, recommendation of ICD implantation is necessary. Through these measures, we may be able to improve the prognosis of these patients (20).

The present study has some limitations. First, the study was conducted retrospectively in a single tertiary center, which might have led to a selection bias and may not be replicable in other centers. Second, the number of patients enrolled in this study was limited. A prospective, multicenter study with a large sample size to confirm the present results is required in the future. Third, none of the patients in our study received an ICD therapy to prevent malignant arrhythmia before and after septal myectomy, which might help to better understand the arrhythmia in these patients. Lastly, our patients did not undergo gene analysis; therefore, we could not study the difference in survival from a genetic standpoint.

\section{Conclusions}

The surgical outcome was poor in a matched cohort of HCM patients with IVST $\geq 30 \mathrm{~mm}$, which was mainly reflected by mortality and the incidence of NYHA class III or IV. In addition, left ventricular end-diastolic diameter and young age were also associated with a poor prognosis in this special HCM population. Further studies on the longterm surgical outcomes of these patients are necessary.

\section{Acknowledgments}

Funding: This work was supported by National Natural Science Foundation of China (Grant No. 81770371).

\section{Footnote}

Reporting Checklist: The authors have completed the STROBE reporting checklist. Available at http://dx. doi. org/10. 21037/jtd-20-1705

Data Sharing Statement: Available at http://dx. doi. org/10. 21037/jtd-20-1705

Conflicts of Interest: All authors have completed the ICMJE uniform disclosure form (available at http://dx. doi. org/10. 21037/jtd-20-1705). The authors have no conflicts of interest to declare.

Ethical Statement: The authors are accountable for all aspects of the work in ensuring that questions related to the accuracy or integrity of any part of the work are appropriately investigated and resolved. The study was conducted in accordance with the Declaration of Helsinki (as revised in 2013). The study was approved by the Institutional Review Board of Anzhen Hospital (Ethic committee study number: $2020034 \mathrm{X}$ ), which is affiliated with Capital Medical University (Beijing, China), and individual consent for this retrospective analysis was waived.

Open Access Statement: This is an Open Access article distributed in accordance with the Creative Commons Attribution-NonCommercial-NoDerivs 4.0 International License (CC BY-NC-ND 4.0), which permits the noncommercial replication and distribution of the article with the strict proviso that no changes or edits are made and the original work is properly cited (including links to both the formal publication through the relevant DOI and the license). See: https://creativecommons.org/licenses/by-nc-nd/4.0/.

\section{References}

1. O'Hanlon R, Grasso A, Roughton M, et al. Prognostic significance of myocardial fibrosis in hypertrophic cardiomyopathy. J Am Coll Cardiol 2010;56:867-74.

2. Hang D, Nguyen A, Schaff HV. Surgical treatment for hypertrophic cardiomyopathy: A historical perspective. Ann Cardiothorac Surg 2017;6:318-28.

3. Ommen SR, Maron BJ, Olivotto I, et al. Long-term effects of surgical septal myectomy on survival in patients with obstructive hypertrophic cardiomyopathy. J Am Coll Cardiol 2005;46:470-6.

4. Authors/Task Force m, Elliott PM, Anastasakis A, et al. 2014 esc guidelines on diagnosis and management of hypertrophic cardiomyopathy: The task force for the diagnosis and management of hypertrophic cardiomyopathy of the european society of cardiology (esc). 
Eur Heart J 2014;35:2733-79.

5. American College of Cardiology Foundation/American Heart Association Task Force on Practice; American Association for Thoracic Surgery; American Society of Echocardiography, et al. 2011 ACCF/AHA guideline for the diagnosis and treatment of hypertrophic cardiomyopathy: a report of the American College of Cardiology Foundation/American Heart Association Task Force on Practice Guidelines. J Thorac Cardiovasc Surg 2011;142:e153-203.

6. Rowin EJ, Maron BJ, Romashko M, et al. Impact of effective management strategies on patients with the most extreme phenotypic expression of hypertrophic cardiomyopathy. Am J Cardiol 2019;124:113-21.

7. Olivotto I, Gistri R, Petrone P, et al. Maximum left ventricular thickness and risk of sudden death in patients with hypertrophic cardiomyopathy. J Am Coll Cardiol 2003;41:315-21.

8. Elliott PM, Gimeno Blanes JR, Mahon NG, et al. Relation between severity of left-ventricular hypertrophy and prognosis in patients with hypertrophic cardiomyopathy. Lancet 2001;357:420-4.

9. Kwon DH, Setser RM, Popovic ZB, et al. Association of myocardial fibrosis, electrocardiography and ventricular tachyarrhythmia in hypertrophic cardiomyopathy: A delayed contrast enhanced mri study. Int J Cardiovasc Imaging 2008;24:617-25.

10. Lai Y, Guo H, Li J, et al. Comparison of surgical results in patients with hypertrophic obstructive cardiomyopathy after classic or modified morrow septal myectomy. Medicine (Baltimore) 2017;96:e9371.

11. Nguyen A, Schaff HV, Nishimura RA, et al. Does septal thickness influence outcome of myectomy for hypertrophic obstructive cardiomyopathy? Eur J Cardiothorac Surg 2018;53:582-9.

Cite this article as: Ren C, Wang S, Yu J, Guo H, Ma H, Wei Z, Liu K, Lai Y. Surgical outcome for hypertrophic cardiomyopathy patients with extreme interventricular septal thickness: a propensity score matched study. J Thorac Dis 2020;12(10):5460-5467. doi: 10.21037/jtd-20-1705
12. Covella M, Rowin EJ, Hill NS, et al. Mechanism of progressive heart failure and significance of pulmonary hypertension in obstructive hypertrophic cardiomyopathy. Circ Heart Fail 2017;10:e003689.

13. Maron BJ, Olivotto I, Spirito P, et al. Epidemiology of hypertrophic cardiomyopathy-related death: Revisited in a large non-referral-based patient population. Circulation 2000;102:858-64.

14. Woo A, Williams WG, Choi R, et al. Clinical and echocardiographic determinants of long-term survival after surgical myectomy in obstructive hypertrophic cardiomyopathy. Circulation 2005;111:2033-41.

15. Desai MY, Bhonsale A, Smedira NG, et al. Predictors of long-term outcomes in symptomatic hypertrophic obstructive cardiomyopathy patients undergoing surgical relief of left ventricular outflow tract obstruction. Circulation 2013;128:209-16.

16. Maron BJ, Savage DD, Wolfson JK, et al. Prognostic significance of 24 hour ambulatory electrocardiographic monitoring in patients with hypertrophic cardiomyopathy: a prospective study. Am J Cardiol 1981;48:252-7.

17. Di Salvo G, Pacileo G, Limongelli G, et al. Non sustained ventricular tachycardia in hypertrophic cardiomyopathy and new ultrasonic derived parameters. J Am Soc Echocardiogr 2010;23:581-90.

18. Monserrat L, Elliott PM, Gimeno JR, et al. Non-sustained ventricular tachycardia in hypertrophic cardiomyopathy. $\mathrm{J}$ Am Coll Cardiol 2003;42:873-9.

19. Geske JB, Ommen SR, Gersh BJ. Hypertrophic cardiomyopathy: Clinical update. JACC Heart Fail 2018;6:364-75.

20. Maron BJ, Rowin EJ, Udelson JE, et al. Clinical spectrum and management of heart failure in hypertrophic cardiomyopathy. JACC Heart Fail 2018;6:353-63. 
Supplementary

Table S1 Relative multivariable imbalance L1 and summary of unbalanced covariates

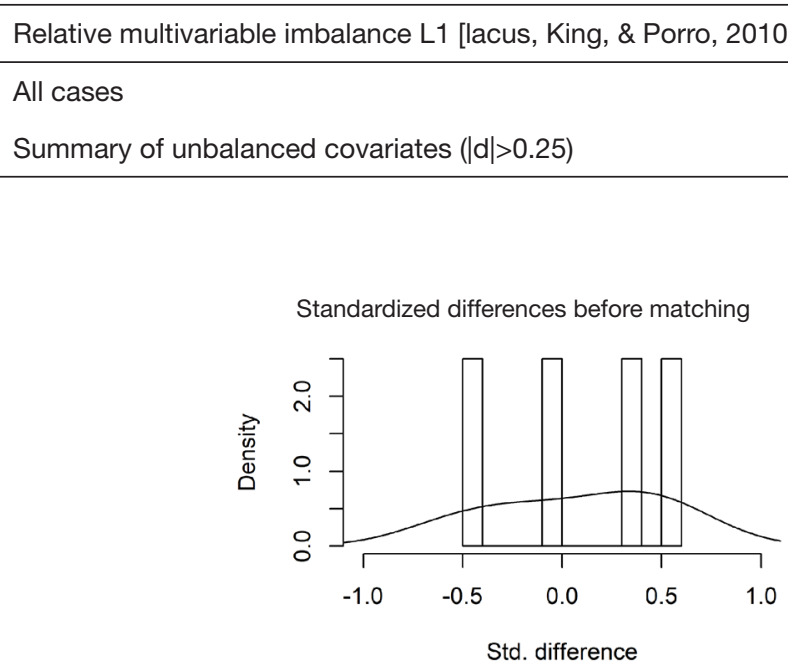

Before matching

After matching

0.675

0.585

No covariate exhibits a large imbalance $(|\mathrm{d}|>0.25)$

Figure S1 Standardized difference before and after matching.

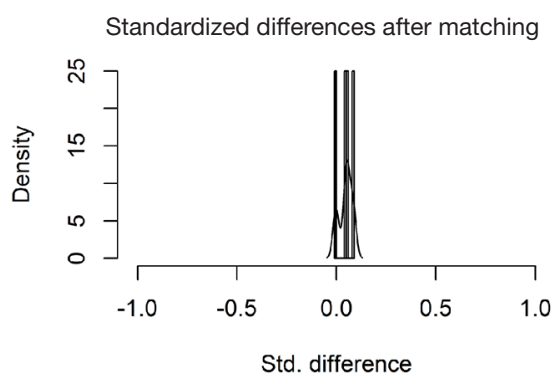

\section{References}

21. Iacus SM, King G, Porro G. Cem: Software for coarsened exact matching. Journal of Statal Software 2009;30. Available online: https://gking.harvard.edu/files/gking/files/jss-paper.pdf 